\title{
Calculations of Cross Sections Data for Scattering of Electrons on $\mathrm{HBr}$
}

\author{
M. Radmilović-Radjenović and Z.Lj. Petrović* \\ Institute of Physics, Pregrevica 118, 11080 Belgrade, Serbia
}

\begin{abstract}
The $R$-matrix method provides a complete theoretical framework for the treatment of low energy electron collisions. The method has been implemented into code Quantemol-N (EE) with a goal to provide users in plasma modeling, swarm studies and other applications with an option to calculate the missing data. In this paper we report on cross sections and rate coefficients for hydrogen bromide obtained by using Quantemol-N. The total cross section has been calculated both with and without Born corrections.
\end{abstract}

PACS numbers: 34.50.-s, 34.50.Gb

\section{Introduction}

Collisions of electrons with atoms and molecules determine the behavior of plasmas. The electron impact ionization of a molecule represents a basic electron-molecule collision process important in many practical applications such as low-temperature processing plasmas [1-3], fusion-edge plasmas $[4,5]$, gas discharges $[6,7]$, radiation chemistry [8], mass spectrometry [9] and chemical analysis [10]. For example, the total cross sections for ionization of molecules by electron impact is one of the essential processes required in the modeling of a wide range of applications, such as plasma processing of semiconductor devices as it defines the number of charged particle balance and sustaining conditions for plasma operation. The importance of electron-molecule collisions has long been recognized and considerable progress in providing experimental data for electron-impact ionization cross sections for a broad range of molecular targets has been achieved in the past decade [11, 12]. Nevertheless, the information on collision data and particularly complete sets of data for most reactive gases used in plasma processing are not available.

Defining low energies as those when projectile electrons have an insufficient energy to ionize the target molecule, the following processes can occur: elastic scattering, electronic excitation, vibrational and rotational excitations, dissociative attachment/recombination and impact dissociation. A common feature of these processes is that they can be considered to proceed via a common intermediary [13]. The $R$-matrix method for treating electron-molecule collisions, used in this article, is designed to obtain accurate wavefunctions for this intermediary and can be used for the modeling the relevant processes. Although the loss of symmetry and increased degrees of freedom mean that electron-molecule collisions are significantly more difficult to treat theoretically than electron-atom collisions, the use of the $R$-matrix method to study

* corresponding author; e-mail: zoran@ipb.ac.rs low-energy electron collisions with molecules is now also well developed. The $R$-matrix method has already been successfully applied to a number of molecules, including $\mathrm{CF}_{3}$ [14], $\mathrm{NH}_{3}[15]$ and $\mathrm{O}_{3}$ [16]. Here we used an implementation developed as user friendly software known as Quantemol-N (EE) developed at the University College London (UCL) [17].

Absolute electron-impact ionization cross sections may be produced from either the Deutsch-Mark (DM) formalism [18] or the Binary-Encounter-Bethe (BEB) theory of Kim and co-workers [19].

Low-pressure plasmas in hydrogen halide-containing gases have been extensively used in various applications such as electron pumped lasers, plasma etching technologies and fabrication of optoelectronic devices [20, 21], thereby dictating the importance of the calculations of cross section data for hydrogen bromide. Transport coefficients for $\mathrm{HBr}$ in dc electric fields, crossed electric and magnetic dc fields and rf fields have already been calculated $[22,23]$. In this work we present our calculations of the total cross section for electron scattering on $\mathrm{HBr}$ molecule and the cross section for ionization based on the BEB theory. In association with cross sections from other sources, we believe that these cross sections would be sufficient to make a reasonable complete set of cross sections for this molecule.

\section{2. $R$-matrix method}

The $R$-matrix method was initially developed by Wigner for the study of nuclear reactions $[24,25]$ and later it was adopted by Burke et al. [26] for the treatment of electron collisions with neutral and ionized atoms [27]. In subsequent years the theory was considerably extended and developed in order to describe a broad range of atomic and molecular continuum processes including data for electron affinities and electron scattering by atoms and molecules, and those for photoionization of atoms and ions.

The essential idea of the method is that configuration space describing the scattered and the target atom or molecule is divided into two regions [12,13]. The internal region, typically of radius $10-20 a_{0}$, where $a_{0}$ represents 
the first Bohr radius, is centered on the center of gravity of the molecule and is assumed to entirely contain the $N$-electron wavefunction of the target molecule. In this region it is necessary to consider all multicentered interactions between the $N+1$ electrons in the system, including exchange. In the inner region, exchange and electron-electron correlations are considerable. As a result, the collision problem within a finite volume can be treated as a molecular bound state problem, by constructing and diagonalizing a Hamiltonian matrix.

On the other hand, in the outer (asymptotic) region, exchange and electron-electron correlations are considered not to be important, and we need to take into account only the long-range multi-polar interactions between the scattering electrons and the target. The problem can be reduced in this region to solving coupled second-order equations, which is in practice done by propagating the $R$-matrix and asymptotic expansion of the solution [12].

The number of configurations that have to be generated in a scattering calculation is the number of target configurations times the number of continuum orbitals summed over all target states. In order to take into account the electron correlation, the target wavefunctions are expanded as a linear combination of configurations, which in turn are constructed in terms of an orthonormal set of molecular orbitals (2). All these steps are now implemented within a package for general purpose calculations, (EE), which was employed here to make the calculations.

\section{Results and discussion}

Calculations have been performed for $\mathrm{HBr}$ by use of Gaussian type basis sets $6-311 \mathrm{G}^{*}$ and DZP. The configuration interaction method was used to construct the molecular (target) ground and electronically-excited state wavefunctions. A number of test calculations were carried out with an increasing number of target electronic states (closed channels) in the close-coupling expansion: 48 and 56 states in both inner and outer regions. The scattering orbitals used by Quantemol-N were those appropriate for radius $20 a_{0}$, where the partial wave expansion includes the g-wave.

The total cross sections calculated using the $R$-matrix method (shown in Fig. 1) represent the sum of elastic and electronically inelastic cross sections, corrected for higher partial waves, using the Born corrections for higher partial waves (solid line), and without Born corrections (dotted line). At higher energies the results of these two methods are in good agreement. The total cross section is dominated by the elastic cross section and the theory provides relatively high values for the elastic cross sections as may be expected for a strongly polar molecule such as $\mathrm{HBr}$.

Apart from the $R$-matrix calculations valid for energies below the threshold for ionization, the code includes an implementation of the BEB technique for determining the ionization cross sections. In Fig. 2 we compare cross sections from ECP [22] (solid curve) and BEB calcula-

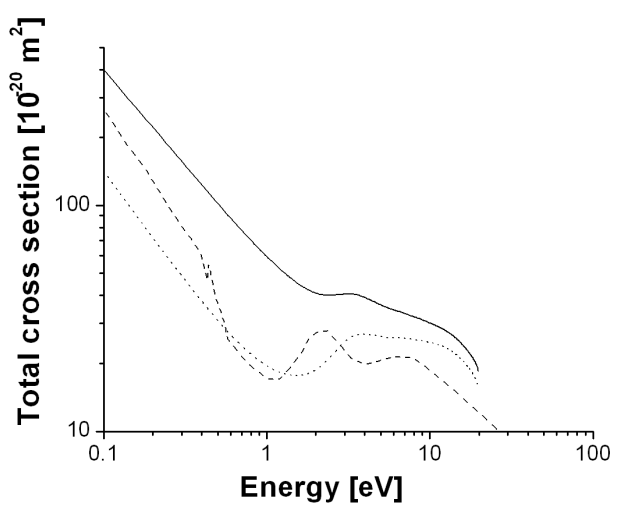

Fig. 1. Total cross section for electron collisions with $\mathrm{HBr}$ molecule: Born corrected cross-section (solid line) and without including Born correlation (dotted line), while the total cross section taken from [28] is shown by dashed line.

tions [19] (dashed and dotted lines) with our Quantemol-N (EE) results (symbols) for present molecule. There is a small discrepancy between BEB and ECP cross section data at low electron energies, while the absolute difference between these results and our calculation is large somewhat below the peak. The descrepency between our data and the results produced by different theoretical methods could be due to the fact that the BEB formalism is very sensitive to the value of the ionisation energy. In our calculations we assume the ionization energy of $11.6 \mathrm{eV}$. For energies higher then $200 \mathrm{eV}$, all methods provide results that differ only by a small percentage. To the best of our knowledge, there are no experimental ionization cross-section data for $\mathrm{HBr}$ in the literature.

Figure 3 illustrates the temperature dependence of the rate coefficients for $\mathrm{HBr}$. For electron mean energies below the threshold, the rate increases rapidly with the temperature (energy). The region where the coefficient reaches the maximum value corresponds to the energy region where the elastic cross section goes through the RT minimum and the vibrational excitation through the maximum.

\section{Conclusion}

We used the Quantemol-N code developed by Tennyson and coworkers [17] which employs the $R$-matrix method to study electron scattering by polyatomic molecules. Calculations were performed for the total (integrated) cross section, ionization cross section as well as for the rate coefficient for the low-energy collisions of electrons with $\mathrm{HBr}$ molecule. Inclusion of the Born corrections (taking into account higher partial waves) in the determination of the total cross section is important at low energies. Results for the cross section were tested for convergence of the polarization interaction by retention of more closed channels in the expansion and the outer region. Results obtained by use of 48 and 56 states appear to be identical and further changes do not appear to be likely. The obtained results for the BEB ionization 


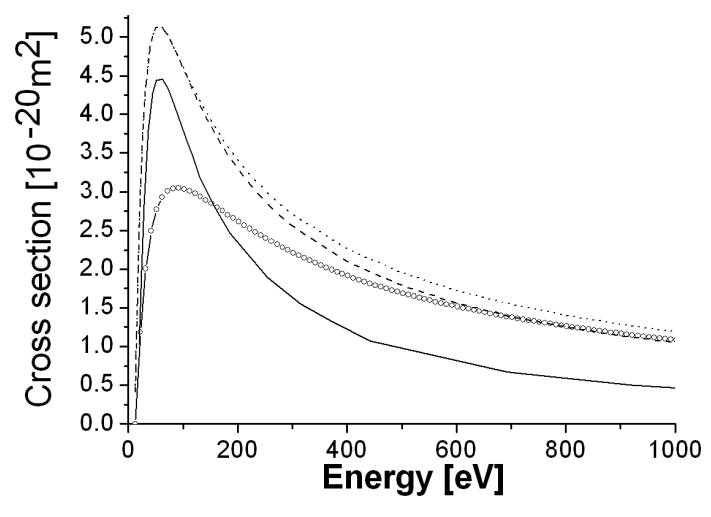

Fig. 2. Ionization cross sections for $\mathrm{HBr}$. Open symbols: our calculations for BEB ionization obtained by use of the (EE) package; solid line: ECP [29] for single ionization; dotted line: Kim and Ali [19] for single ionization; dashed line: Kim and Ali [19] for counting ionization.

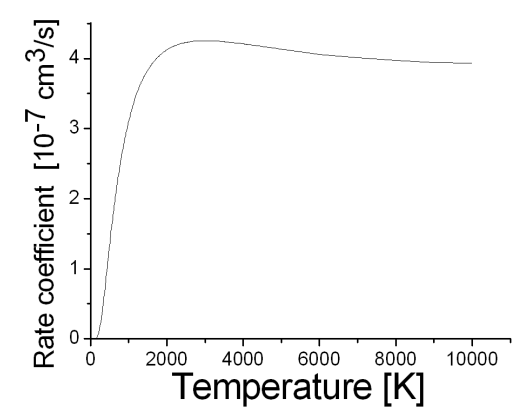

Fig. 3. The rate coefficient versus temperature for $\mathrm{HBr}$.

differ from other sources but it was not possible to check the input data used in those calcualtions. Calculated data presented in this work, particularly the total cross section will enable completion of a complete set of input data for $\mathrm{HBr}$ and more accurate calculations of transport coefficients for this molecule for which there is a lack of experimental data.

\section{Acknowledgments}

The authors acknowledge financial support of the Ministry of Science of the Republic of Serbia 141025. Authors are grateful to J. Tennyson, H.N. Vahrambia, D.N. Brown, J.J. Munro, I. Rozum and N. Vinci, for providing us with proper basis sets and upgrading the Quantemol code to suit this problem.

\section{References}

[1] C. Winstead, V. McKoy, Adv. Atom. Mol. Opt. Phys. 43, 111 (2000).

[2] M. Kimura, Electron Collisions with Molecules in gases: Applications to Plasma, Academic Press, London 2001.

[3] L.G. Christophorou, J.K. Olthoff, Fundamental electron interactions with plasma processing gases, Kluwer Academic/Plenum Publisher, New York 2004.
[4] J. Schweinzer, Atomic and molecular processes in $\mathrm{fu}$ sion edge plasmas, Plenum, New York 1995.

[5] R.K. Janev, Collision Processes of Atomic and Molecular Hydrogen in Fusion Plasmas: The Cross-Section Data Status, Springer Berlin Heidelberg 2006.

[6] M.A. Biondi, Appl. Spectrosc. 11, 119 (1957).

[7] D. Marić, P. Hartmann, G. Malović, Z. Donkó, Z.Lj. Petrović, J. Phys. D, Appl. Phys. 36, 2639 (2003).

[8] O. Šašić, Z.Lj. Petrović, Rad. Phs. Chem. 76, 573 (2007).

[9] U. Onthong, H. Deutsch, K. Becker, S. Matt, M. Probst, T.D. Märk, Inter. Journal of Mass Spectrometry 214, 53 (2002).

[10] M.J. Fitch, M.R. Leahy-Hoppa, E.W. Ott, R. Osiander, Chem. Phys. Lett. 443, 284 (2007).

[11] W.M. Huo, F.A. Gianturco, F.A. (Eds.), "Computational Methods for Electron Molecule Collisions" (Plenum Press, New York 1995).

[12] L.A. Morgan, J. Tennyson, C.J. Gillan, Comput. Phys. Commun. 114, 120 (1998).

[13] J. Tennyson, J.D. Gorfinkiel, I. Rozum, C.S. Trevisan, N. Vinci, Radiation Phys. Chemistry 68, 65 (2003).

[14] I. Rozum, N.J. Mason, J. Tennyson, New J. Phys. 5, 155 (2003).

[15] H. Mujal, K.L. Baluja, Phys. Rev. A 74, 032712 (2006).

[16] M. Gupta, K.L. Baluja, J. Phys. B, At. Mol. Opt. Phys. A 395, 195 (2005).

[17] J. Tennyson, D.B. Brown, J.J. Munro, I. Rozum, H.N. Varambhia, N. Vinci, J. Phys., Conference Series 86, 012001 (2007).

[18] M. Probst, H. Deutsch, K. Becker, T. D. Märkde, International Journal of Mass Spectrometry 206, 31 (2001).

[19] M.A. Ali, Y.K. Kim, J. Phys. B, At. Mol. Opt. Phys. 41, 145202 (2008).

[20] T.D. Bestwick, G.S. Oehrlein, Y. Zhang, G.M.W. Kroesen, E. de Frésart, Appl. Phys. Lett. 59, 336 (1991).

[21] K.M. Chang, T.H. Yeh, I.C. Deng, H.C. Lin, J. Appl. Phys. 80, 3048 (1996).

[22] N. Nakano, N. Shimura, Z.Lj. Petrović, T. Makabe, Phys. Rev. E 49, 4455 (1994).

[23] R.E. Robson, R.D. White, Z.Lj. Petrović, Rev. Mod. Phys. 77, 1303 (2005).

[24] E.P. Wigner, Phys. Rev. 70, 15 (1946).

[25] A.M. Lane, R.G. Thomas, Rev. Mod. Phys. 30, 257 (1958).

[26] M LeDourneuf, B.I. Schneider, P.G. Burke, J. Phys. B, At. Mol. Phys. 12, L365 (1979).

[27] P.G. Burke, K.A. Berrington, Atomic and Molecular Processes, an R-matrix Approach, Institute of Physics Publishing, Bristol, 1993.

[28] O. Šašić, S. Dujko, Z.Lj. Petrović, T. Makabe, Jpn. J. Appl. Phys. 46, 3560 (2007).

[29] W.M. Huo, Y.K. Kim, Chem. Phys. Lett. 319, 576 (2000). 